\title{
CONSIDERATIONS REGARDING ANODONTIA IN DENTAL MEDICAL PRACTICE
}

\section{Iordan Dumitru Andreea Dona1', Kozma Andreī2, Bodnar Gristina33, Agop-Forna Doriana}

1 "Titu Maiorescu" University, Bucharest

2 "Alessandrescu Rusescu" National Institute for Mother and Child Health, Bucharest

3 "Carol Davila" University of Medicine and Pharmacy, Bucharest - Faculty of Dental Medicine

4"Grigore T.Popa" Univesity, Iasi

\begin{abstract}
Aim: This article is set on presenting various types of anodontia, the most frequent types found in a dental office and to bring awareness to parents and also the dentists on the signs of agenesis and its consequences in time. Material and method: for a year (1st September 2017 - 31th August 2018) various patients with different types and locations of anodontia were diagnosed correctly by an oral and radiological exam in a dental office. Results. Anodontia is found almost equally in both sexes without major differences between males and females. Real agenesis is most frequently found in the second premolars - study reports show that this is because of phylogenetic evolution. False agenesis is more frequent in the 3rd molars because they are most likely extracted. Third molars are usually extracted because they don thave space to emerge on the maxilla or mandible or they emerge in a vicious position. Canine agenesis has a very low frequency. Conclusions: It is very important to do a thorough check-up and a correct case history followed by radiological investigations each time a suspicion of a possible anodontia exists. It can only be certainly diagnosed by having a full oral and radiological exam. Also a difference must be made between the real, false and pseudoanaodontia.
\end{abstract}

Keywords: anodontia, agenesis, oral exam, radiological exam

\section{Introduction}

Dental abnormalities include a large specter of aspects in the dental literature; therefore they are widely classified and variously defined.

The abnormality is in general the result of a disruption that interests the formation period of the dental lamina and / or the dental buds. It can affect both temporary and deciduous dentition. Patients with these kinds of abnormalities have a low density bone that represents a real challenge for dental treatments.

Agenesis can appear isolated or within the pale of other genetic disorders.

These dental abnormalities of growth are different of the abnormalities that appear as a consequence of some etiological factors regarding the functioning tooth. Dental growth abnormalities are the result of genetic factors or the actions of local or general environmental factors or a combination of these two factors.

By some authors anodontia is a phylogenetic growth abnormality only present in symmetrical teeth.

It must be differentiated by the false and pseudo agenesis.

Pseudo agenesis or dental inclusion is characterized by the clinical absence of teeth because of dental inclusions or late eruption.

False agenesis involves the clinical absence 
Dental abnormalities include a large specter of aspects in the dental literature; therefore they are widely classified and variously defined.

The abnormality is in general the result of a disruption that interests the formation period of the dental lamina and / or the dental buds. It can affect both temporary and deciduous dentition. Patients with these kinds of abnormalities have a low density bone that represents a real challenge for dental treatments.

Agenesis can appear isolated or within the pale of other genetic disorders.

These dental abnormalities of growth are different of the abnormalities that appear as a consequence of some etiological factors regarding the functioning tooth. Dental growth abnormalities are the result of genetic factors or the actions of local or general environmental factors or a combination of these two factors.

By some authors anodontia is a phylogenetic growth abnormality only present in symmetrical teeth.

It must be differentiated by the false and pseudo agenesis.

Pseudo agenesis or dental inclusion is characterized by the clinical absence of teeth because of dental inclusions or late eruption.

False agenesis involves the clinical absence of teeth by cause of natural exfoliation or by removal of the bud of the successor permanent tooth while extracting the temporary tooth.

\section{Material and method}

At a dental office situated in the rural area near Bucharest, in a year's time, from 1st September 2017 and 30th august 2018, a number of 687 patients were investigated. A total of $11.06 \%$ (76 patients) were diagnosed with anodontia.

The diagnostic was made by a thorough case history and intraoral examination, but the radiological examination offered the certainty of such a diagnostic. It is important when in doubt that an agenesis is present to do a thorough check up and a radiological exam. It can be certainly diagnosed only by radiological and clinical examination.

\section{Case 1}

A 23 years old patient (Figure 1), female, is presented at the dental office for a dental cleaning. After a simple oral exam it is observed that on the left inferior arch 8.5 is still present and 4.5 is absent. In the 3 th quadrant no such alterations exist.

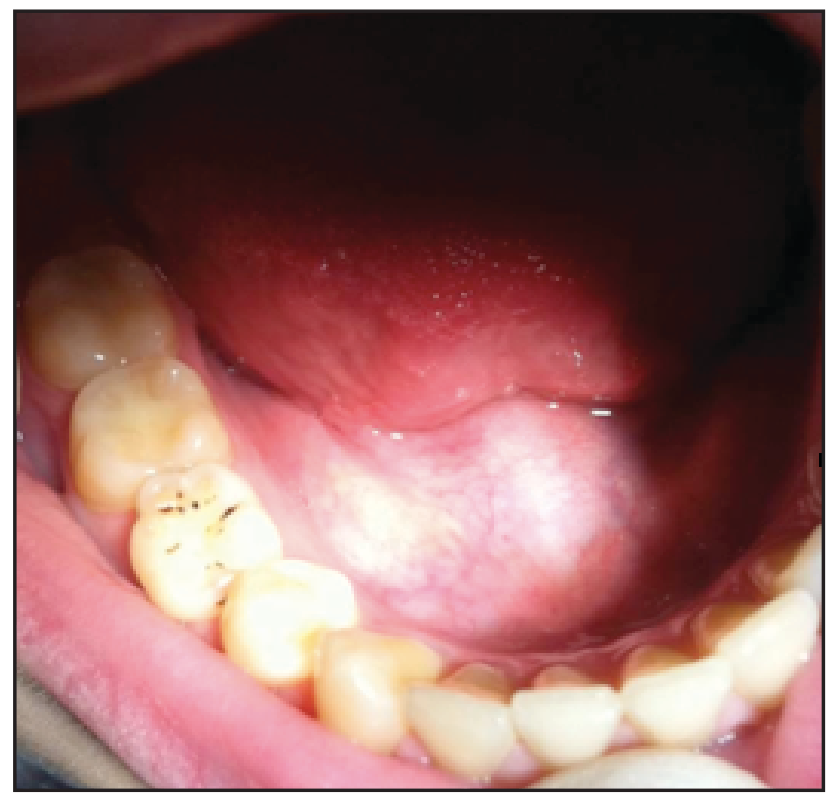

Figure 1. V. R., female 23 years old - 4.5 Anodontia with the persistence of 8.5

After the case history and oral exam it is discovered that the patient had no idea of this situation. In addition, she has missing 3rd molars. A radiological exam is recommended. Teeth 3.8 and 4.8 are missing because they were extracted for orthodontic purpose in the past and can lead us to a false anodontia diagnostic. Conservatory treatment of the temporary tooth is indicated in this case, in order to keep the tooth functional as much time as possible.

\section{Case 2}

A 23 years old patient (Figure 2), female, is presented at the dental office for a dental cleaning. After a simple oral exam it is observed that on the left inferior arch 7.5 is still present and 3.5 is absent. In the 4th quadrant no such alterations exist. After the case history and oral exam it is discovered that the patient had no idea of this situation. Also she has missing 3rd molars.

A radiological exam is recommended for a 
certainty diagnostic. A good hygiene is required in order to keep the temporary tooth functional and cavity-free. In this case an enameloplasty is required to appreciate the depths of the carious lesion. Regarding the depth the filling will be executed and the patient will be made aware of his abnormality.

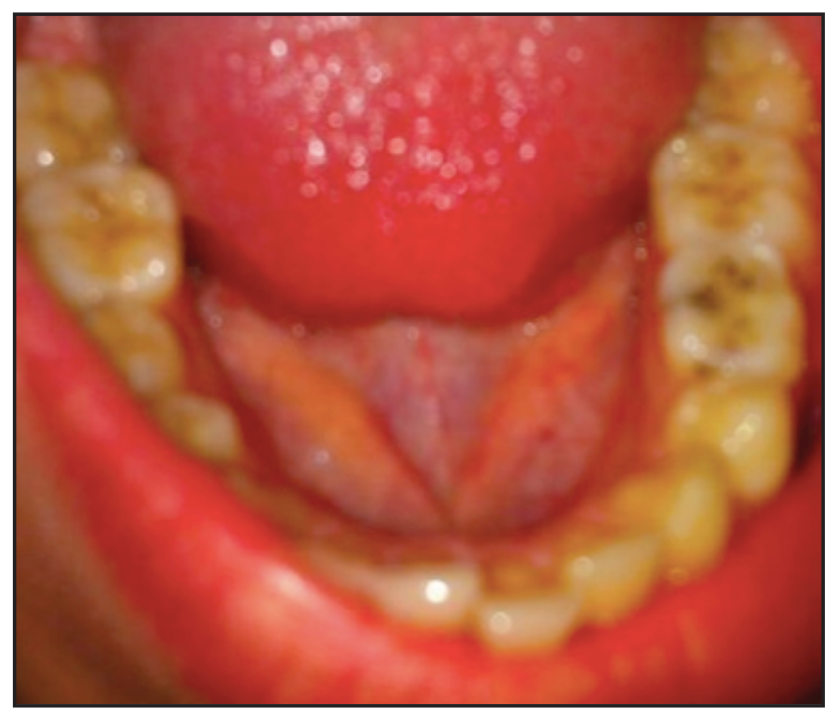

Figure 2 P.M., female 23 years old - 3.5 Anodontia with the persistence of 7.5

\section{Case 3}

A 5 years old patient, male is presented in the dental office for a check-up. After the radiological exam it is diagnosed that teeth 4.5 and 3.5 are missing so this is a case of agenesis and the 3rd molars are not yet observed intraosseous. (Figure 3). It is recommended to treat the cavities found in the 2nd temporary molars 8.5 and 7.5 in order to keep them functional on the dental arch.

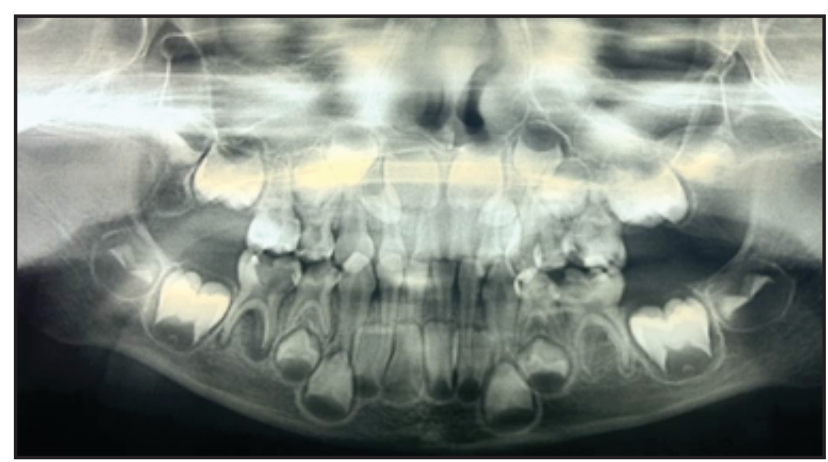

Figure 3. - P.B., male- Hypodontia in 3.5 and $4.5-$ radiologic aspect at 5 1/2 years.

Tooth 8.5 has an extensive carious lesion with endodontic lesions and tooth 7.5 has an extensive carious lesion that can lead to the need of endodontic treatment therefore the vitality of 7.5 must be kept in order to prevent other complications.

\section{Case 4}

A 9 years old patient, male, (Figure 4) is presented at the dental office for a regular check up.

Agenesis of teeth 3.5 and 4.5 is observed which is similar to his cousin presented in case 3 above. The 3rd molars are looming $(1.8,2.8,3.8$, and 4.8).

From the case history it is disclosed that the paternal grandmother had the same abnormality. More so it is observed that a big inconsistency between the biological age and teeth age exists.

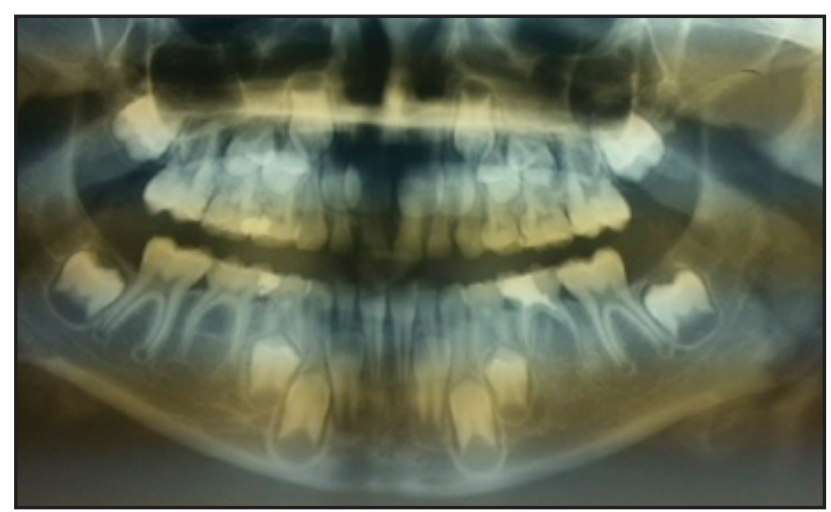

Figure 4 P.R. male- Hypodontia in 3.5 and $4.5-$ radiologic aspect at 9 years old

\section{Case 5}

An 8 years old patient, female, with incontinentia pigmenti diagnostic is presented in the dental office for a checkup. At the oral examination all the permanent teeth in the right mandible are absent and on the left only teeth 3.2, 3.6. 3.7 are present, from which, only tooth 3.6 has emerged. In the maxilla the permanent dentition is not as severely affected as the mandible teeth with only missing $1.2,2.1,2.2$, and 2.7 (Figure 5).

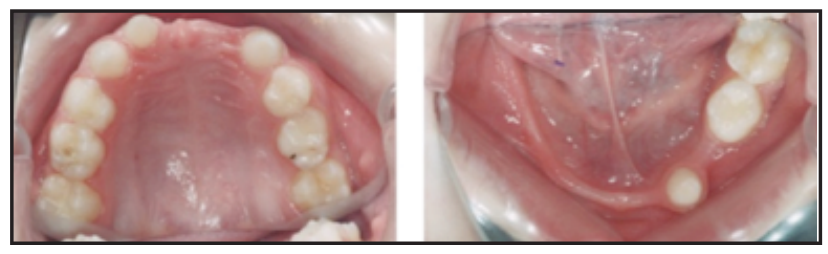

Figure 5. R. M. 8 years old-a) maxilla; b) mandible 
By connecting the clinical exam with the radiological one it is clear that both dentitions are affected and it is very possible that in deciduous dentition all the buds of right mandible teeth might have been missing and also the buds of 71, 72,74 and the maxilla buds of $51,61,62$. Tooth 3.4 might exist (Figure 6)

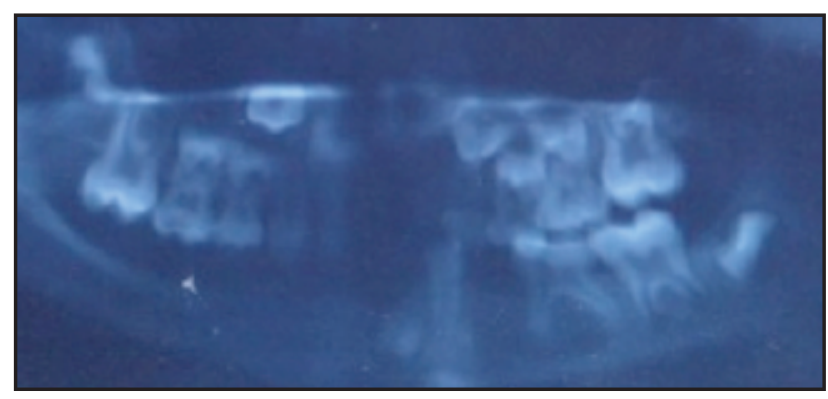

Figure 6 R.M. 8 years old-radiological aspect

\section{Case 6}

A 7 years old patient, male, with ectodermal displasia is presented at the dental office with only 1.1 and 2.1 present in the oral cavity. (Figure 7)

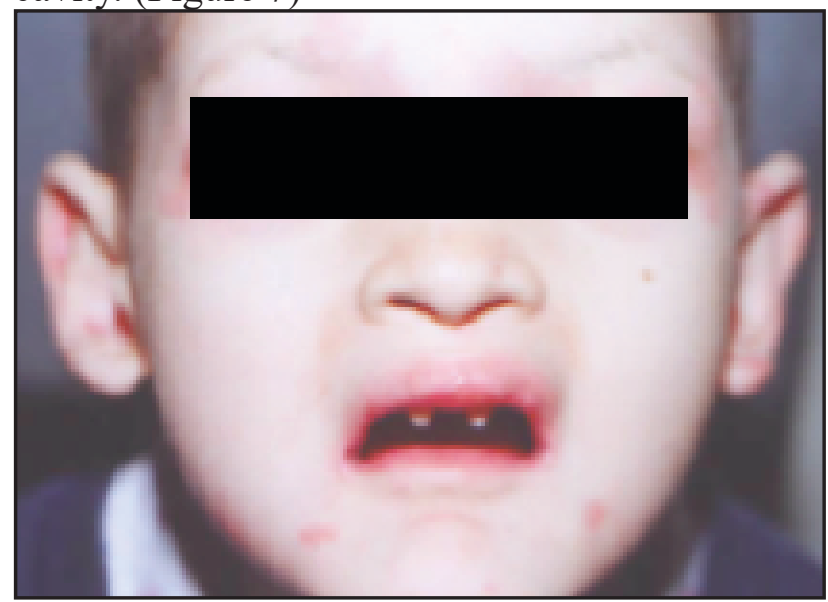

Figure 7 - A.C. male, anhidrotic ectodermal displasia . Agenesis of all teeth except 1.1 and 2.1 .

The radiological exam doesn't show any other existing teeth. There is a possibility that the agenesis was present also in the deciduous dentition, beeing part of the genetic disorder

The only available treatment that can assure a functional and psychical confort was the realisation of dentures adapted to his age and without endangering the normal growth of the maxilla and mandible. (Figure 8)

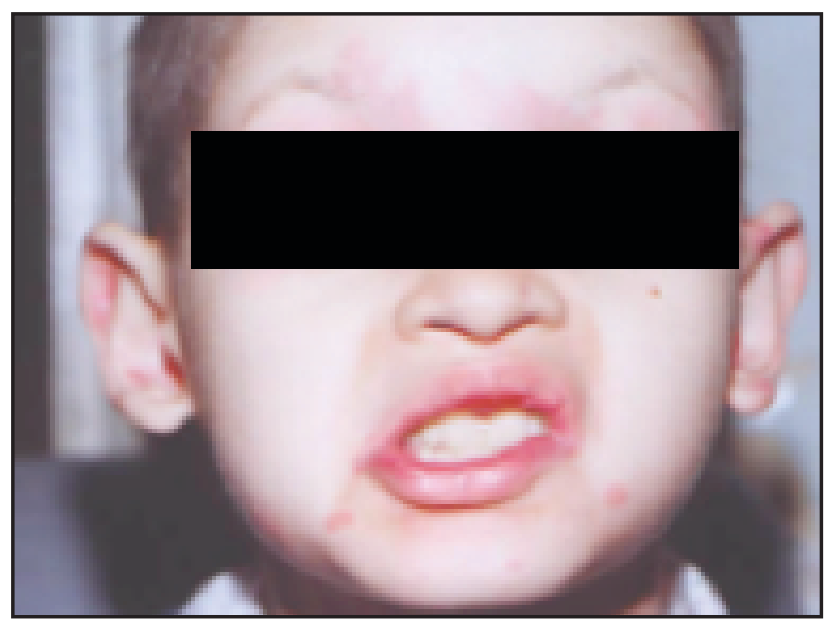

Figure 8 A.C. ,male, anhidrotic ectodermal displasia, patient with dentures

\section{Case 7}

A 17 years old patient, male (Figure 9) is presented for a dental cleaning. After the oral exam, it is aquired that tooth 5.3 is still present on the upper arch and is smaller by $30 \%$ than his similar 2.3. The cusp is deeply abraded therefore it indicates it is a temporary tooth.

The canine anodontia, even though a very rare diagnostic, was made after the radiological exam. (Figure 10). It is recommended to keep a very good oral hygiene and at the first enamel affliction the patient is to be presented in the dental office for a conservatory treatment.

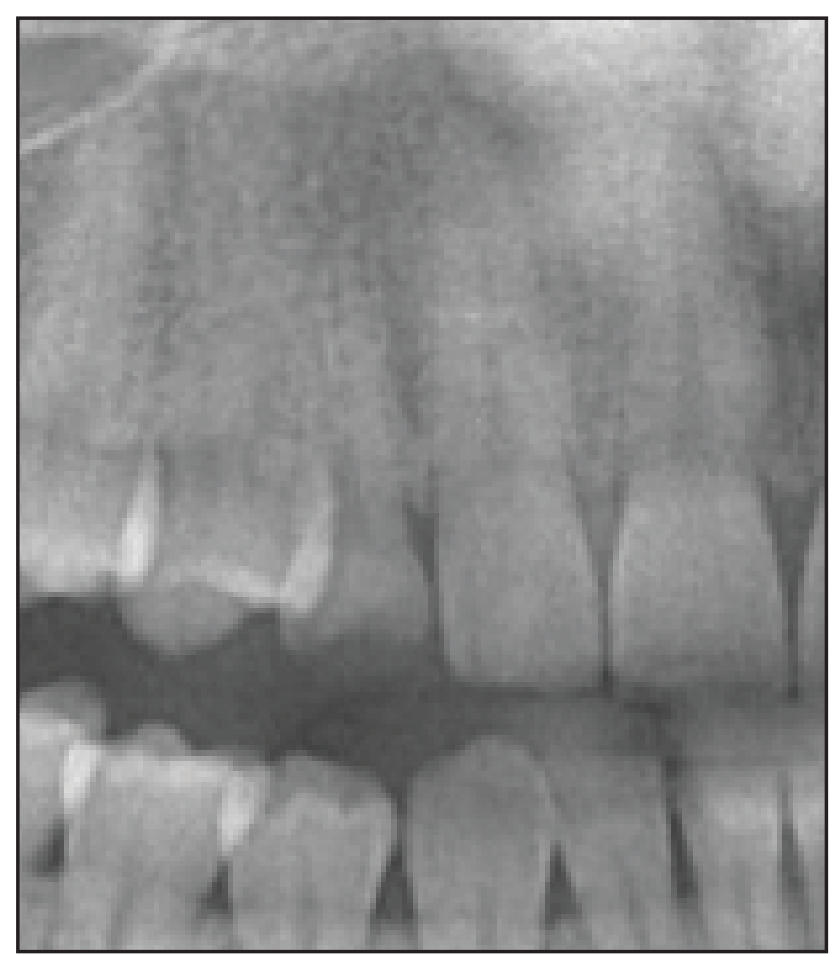

Figure 9 C.C., male-radiologic aspect 


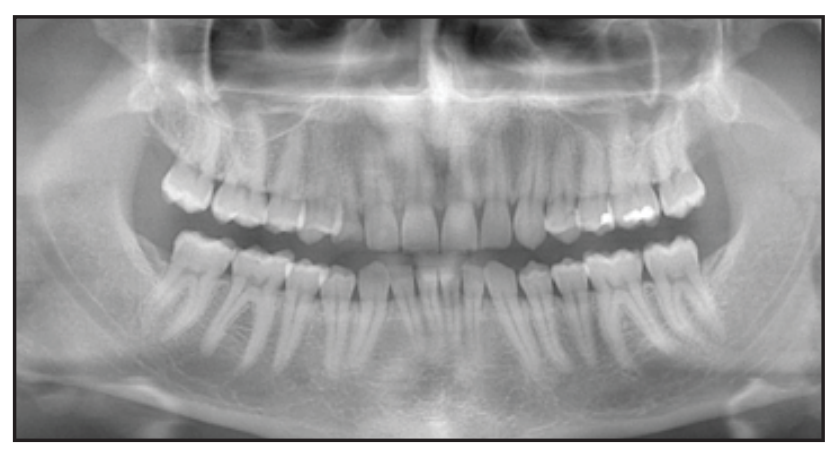

Figure 10 C.C., male-aspect radiological aspect

\section{Discussions}

The difference between real, false and pseudoanodontia must be made. Regarding the type of agenesis the right treatment can be decided in order to successfully solve the case. It can only be certainly diagnosed by having a full oral and radiological exam.

False anodontia is frequent in the 3rd molars when they are extracted because the arches are too small and there is no space for them to erupt or if they are situated in various intraosseous positions.

Real agenesis is most frequent in the 2nd premolars - study reports show that this is a phylogenetical evolution. Canine agenesis is relatively rare.

\section{Conclusions:}

Anodontia is found in both sexes almost equally without major differences.

It is very important when there is a doubt of anodontia to do a thorough checkup and a serious case history followed by the adequate radiological examination each time a suspicion of a possible anodontia exists - only on the radiological examination we can certainly diagnose anodontia.

Also a difference must be made between the real, false and pseudoanaodontia.

\section{Compliance with Ethics Requirements:}

„The authors declare no conflict of interest regarding this article“

„The authors declare that all the procedures and experiments of this study respect the ethical standards in the Helsinki Declaration of 1975, as revised in 2008(5), as well as the national law. Informed consent was obtained from the patient included in the study“

„No funding for this study“

\section{References}

1. Luca R. Pedodontie Vol III. Bucuresti: Cermaprint; 2013. 190 p.

2. Laskaris G. Color Atlas of Oral Diseases in Children and Adolescents: Thieme Medical Publishers; 2000.

3. Borzabadi-Farahani A. Orthodontic considerations in restorative management of hypodontia patients with endosseous implants. J Oral Implantol. 2012;38(6):77991.

4. Yap AK, Klineberg I. Dental implants in patients with ectodermal dysplasia and tooth agenesis: a critical review of the literature. Int J Prosthodont. 2009;22(3):268-76

5. Ionescu E. Anomaliile dentare de numar. Bucuresti: Cermaprint; 2000. 140 p

6. Iordan-Dumitru AD, Luca R. Anomalii dentare de dezvoltare la un copil cu incontinentia pigmenti. Prezentare de caz. Romanian Journal of stomatology. 2013; $\operatorname{LIX}(4 / 20)$. 